\title{
Transport coefficients of a massive pion gas
}

\author{
D. Fernández-Fraile ${ }^{1 ; 1)} \quad$ A. Gómez Nicola ${ }^{2 ; 2)}$ \\ 1 (Institut für Theoretische Physik, Johann Wolfgang Goethe-Universität, Max-von-Laue-Str. 1, 60438 Frankfurt am Main, \\ Germany) \\ 2 (Departamento de Física Teórica II, Universidad Complutense, 28040 Madrid, Spain)
}

\begin{abstract}
We review or main results concerning the transport coefficients of a light meson gas, in particular we focus on the case of a massive pion gas. Leading order results according to the chiral power-counting are presented for the DC electrical conductivity, thermal conductivity, shear viscosity, and bulk viscosity. We also comment on the possible correlation between the bulk viscosity and the trace anomaly in QCD, as well as the relation between unitarity and a minimum of the quotient $\eta / s$ near the phase transition.
\end{abstract}

Key words Transport coefficients, Heavy-ion collisions, Chiral Perturbation Theory.

PACS 11.10.Wx, 12.39.Fe, 25.75.-q

\section{Introduction}

The calculation of transport coefficients in quantum field theory at intermediate and strong coupling is still a challenge from both the analytical and the numerical points of view. Due to their intrinsic nonperturbative nature, even in weakly interacting theories a resummation of an infinite number of diagrams is needed in order to obtain the leading-order result. In the strongly coupled regime, the only reliable method available is the AdS/CFT correspondence ${ }^{1}$, although it is only applicable to a limited class of field theories. On the other hand, lattice techniques for extracting these quantities are still in their infancy, and the inclusion of a finite quark chemical potential in these calculations makes of it an even more difficult challenge because of the sign problem ${ }^{2}$.

Transport coefficients are essential inputs to describe the evolution of systems not far from equilibrium. In particular, during the last years there has been a very active effort to analyze them from both the theoretical and phenomenological points of view in the context of heavy-ion collisions, condensed matter physics, astrophysics and cosmology. We will be here interested in QCD at zero baryon chemical potential and temperatures below the chiral phase transition, where the hadrons are the relevant degrees of freedom. Near $T_{\mathrm{c}}$ the QCD coupling constant is not small, and usual perturbative techniques give in prin- ciple not reliable results there. We will present results concerning a diagrammatic calculation of transport coefficients in the context of Chiral Perturbation Theory for a pion gas, which is the dominant component of the hadron gas at low temperatures, and we will extrapolate these results up to temperatures close to $T_{\mathrm{c}}$ by using unitarization methods.

\section{Transport coefficients}

Consider a quantum field characterized by two conserved currents, the energy-momentum tensor $T_{\mu \nu}$ and another current $N_{\mu}$. In the local rest frame of the quantum fluid, we can derive two constitutive relations by considering a small departure from a reference equilibrium state:

$$
\begin{aligned}
& \left\langle\hat{T}_{i j}\right\rangle=P_{\mathrm{eq}} \delta_{i j}+\eta\left(\partial_{i} U_{j}+\partial_{j} U_{i}+\frac{2}{3} \delta_{i j} \partial_{k} U^{k}\right)-\zeta \delta_{i j} \partial_{k} U^{k}, \\
& \left\langle\hat{T}^{i 0}\right\rangle-h\left\langle\hat{N}^{i}\right\rangle=\kappa \frac{T^{2}}{h} \partial_{i}\left(\frac{\mu}{T}\right),
\end{aligned}
$$

where $\langle\cdot\rangle$ denotes a non-equilibrium statistical average, $U^{\mu}$ is the fluid velocity normalized as $U^{2}=1$, $P_{\text {eq }} \equiv\left\langle-\hat{T}_{k}^{k} / 3\right\rangle_{\text {eq }}$ is the (equilibrium) pressure, which satisfies an equation of state $P_{\text {eq }}=P_{\text {eq }}(\epsilon, n)$ in terms of the energy density $\epsilon \equiv\left\langle\hat{T}_{00}\right\rangle$ and the charge density $n \equiv\left\langle\hat{N}^{0}\right\rangle$, and $h=(\epsilon+P) / n$ is the enthalpy. The shear viscosity $\eta$, the bulk viscosity $\zeta$, and the thermal conductivity $\kappa$, determine the departure from the

Received 15 December 2009

1) E-mail:danfer@th.physik.uni-frankfurt.de

2) E-mail: gomez@fis.ucm.es 
equilibrium state. On the other hand, if we consider an electrically charged field, the DC conductivity $\sigma$ is defined through the constitutive relation $\left\langle\hat{J}^{i}\right\rangle=\sigma E^{i}$, where $\left\langle\hat{J}^{i}\right\rangle$ is the electric current produced after applying a (classical) constant electric field $E^{i}$ to the system.

From (1) we see that the shear viscosity is related to departures from equilibrium due to transverse flow of momentum, whereas the bulk viscosity is related to variations of pressure, energy density, and charge density due to uniform compression or expansion of the system, because $\Delta P-c_{\epsilon} \Delta \epsilon-c_{n} \Delta n=-\zeta \nabla \cdot \boldsymbol{U}$, where $\Delta P \equiv\left\langle-T_{k}^{k} / 3\right\rangle-P_{\mathrm{eq}}\left(\epsilon_{0}, n_{0}\right), c_{\epsilon} \equiv\left(\partial P_{\mathrm{eq}} / \partial \epsilon\right)\left(\epsilon_{0}, n_{0}\right)$, $c_{n} \equiv\left(\partial P_{\text {eq }} / \partial n\right)\left(\epsilon_{0}, n_{0}\right), \Delta \epsilon \equiv \epsilon-\epsilon_{0}$, and $\Delta n \equiv n-n_{0}$, with $\epsilon_{0}$ and $n_{0}$ the values corresponding to the reference equilibrium state. And from (2) we see that the thermal conductivity is identically zero if there is not a conserved current in the system besides the energy-momentum tensor (in the case of the pion gas we consider that the conserved charge is the total number of pions ${ }^{3}$ ).

In Linear Response Theory (LRT), a transport coefficient $\mathcal{T}$ is expressed in terms of retarded correlation functions through the Kubo formulas:

$$
\begin{aligned}
& \mathcal{T}=C_{\mathcal{T}} \lim _{\omega \rightarrow 0^{+}} \lim _{p \rightarrow 0^{+}} \frac{\partial \rho_{\mathcal{T}}(\omega, p)}{\partial \omega}, \\
& \rho_{\mathcal{T}}(\omega, p)=2 \operatorname{Imi} \int \mathrm{d}^{4} x \mathrm{e}^{\mathrm{i} p \cdot x} \theta(t)\left\langle\left[\hat{\mathcal{S}}_{\mathcal{T}}^{\alpha}(x), \hat{\mathcal{S}}_{\alpha}^{\mathcal{T}}(0)\right]\right\rangle_{\text {eq }}
\end{aligned}
$$

where $\alpha$ represents some set of indices. For the different transport coefficients considered here ${ }^{3} *$.

$$
\begin{aligned}
& C_{\sigma} \equiv-\frac{1}{6}, C_{\eta} \equiv \frac{1}{20}, C_{\zeta} \equiv \frac{1}{2}, C_{\kappa} \equiv-\frac{1}{6 T}, \\
& \hat{\mathcal{S}}_{\sigma}^{i} \equiv \hat{J}^{i}, \\
& \hat{\mathcal{S}}_{\eta}^{i j} \equiv \hat{T}^{i j}-g^{i j} \hat{T}_{k}^{k} / 3, \\
& \hat{\mathcal{S}}_{\zeta} \equiv-\hat{T}_{k}^{k} / 3-c_{\epsilon} \hat{T}^{00}-c_{n} \hat{N}^{0}, \\
& \hat{\mathcal{S}}_{\kappa}^{i} \equiv \hat{T}^{0 i}-h \hat{N}^{i} .
\end{aligned}
$$

\section{$2.1 \quad$ Diagrams vs. kinetic theory}

Due to the zero momentum limit, the leadingorder contribution (in powers of the coupling constant) to transport coefficients obtained by using the formula (3) involves the resummation of an infinite number of ladder diagrams, as was first shown by Jeon and Yaffe ${ }^{[5}$ in the case of a $\lambda \phi^{4}$ theory. This is essentially because every two propagators which share the same four-momentum in that limit give a contribution $\propto 1 / \lambda^{2}$. In the case of the bulk viscosity, in addition, another set of diagrams called chain (or bubble) diagrams give an important vertex correction that has to be taken into account. In those papers it was also proved that, in the weakly coupled regime, this resummation is equivalent to solving the Boltzmann equation corresponding to an effective kinetic theory where thermal effects are incorporated into the dispersion relation of the quasiparticles as well as into their scattering amplitudes. Consequently, it is in principle more convenient technically to adopt the effective kinetic theory approach to calculate transport coefficients in weakly coupled theories 6 7. However, in the context of ChPT where we have carried out our analysis for the pion gas, the relevant power-counting for estimating the contribution from the different Feynman diagrams suggests that the leading order of transport coefficients is given by a one-loop diagram with dressed pion propagators 3] 8 , which simplifies significantly the calculation.

\subsection{Results for a pion gas}

We dress the thermal pion propagators by considering the lowest order process according to ChPT in pion-pion scattering, and we unitarize the corresponding amplitude by the Inverse Amplitude Method (IAM) 3 9, (the ChPT expansion eventually violates unitarity as we increase the collision energy). Unitarization allows to extend the range of applicability of the ChPT expansion to higher temperatures, and correctly reproduces the resonances $f_{0}(600)$ and $\rho(770)$ in the pion-pion scattering channels $(I, J)=(0,0),(1,1)$ respectively. This unitarization also allows the study of the evolution of these resonances with temperature and baryon density, as was presented in the references 910 .

In Fig. 1 we can see that unitarization changes the qualitative behavior of transport coefficients with temperature. In particular it makes the quotient $\eta / s$, with $s$ the entropy density, to satisfy the conjectured lower bound $1 / 4 \pi$ from $\mathrm{AdS} / \mathrm{CFT}^{1}$, and the value near $T_{\mathrm{c}}$ is close to the full hadron resonance + Hagedorn states gas result 12 . In the case of the bulk viscosity, unitarization enhances the anomalous peak close to $T_{\mathrm{c}}{ }^{13}$, nevertheless the quotient $\zeta / s$ is still smaller than $\eta / s$, although vertex corrections in the case of $\zeta$ may play an important role 4 14.

${ }^{*}$ There is typo in the formula (3.43) of Ref. ${ }^{3}$, where $c_{s}^{2}$ and $\mu$ should be substituted by $c_{\epsilon}$ and $c_{n}$ respectively. 

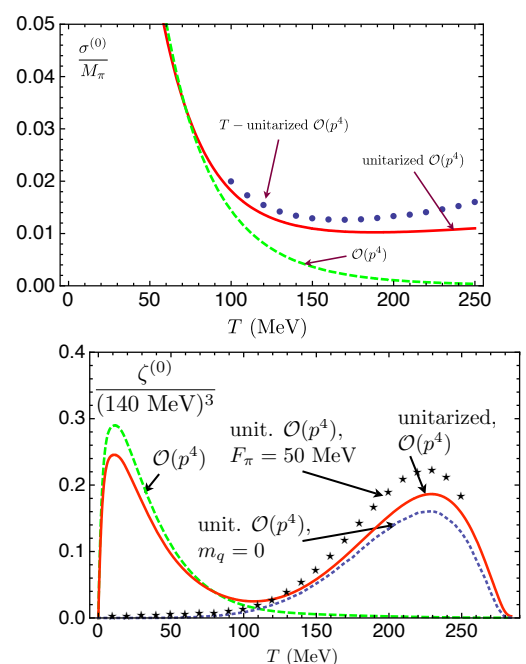
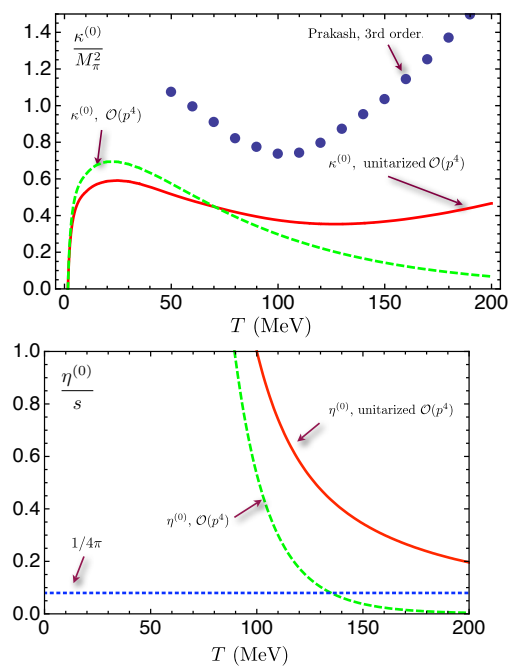
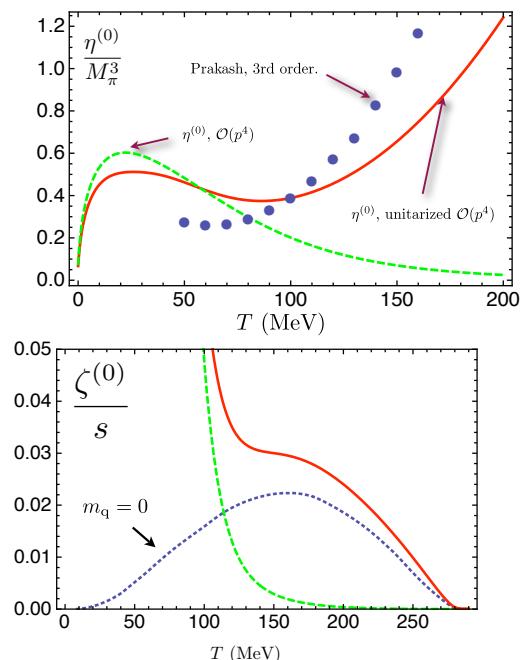

Fig. 1. Leading order contribution (one loop) to the transport coefficients for a massive pion gas according to ChPT. The solid red line represents the unitarized result to $\mathcal{O}\left(p^{4}\right)$ in the pion scattering amplitudes. The dashed green line represents the non-unitarized result to $\mathcal{O}\left(p^{4}\right)$. For the thermal conductivity and the shear viscosity we compare with the kinetic theory results of [11].

It has been recently proposed using a sum rule and an ansatz for the spectral function that the bulk viscosity and the trace anomaly in QCD should be correlated ${ }^{[15}$, so that a maximum in the trace anomaly would drive a maximum in $\zeta$. The spectral function was modified very recently in $\frac{16}{16}$, and the form of the ansatz has been questioned in 17 . So it is at the moment not clear how these two quantities are actually related to each other. According to our results for the trace anomaly in Fig. 2 and the bulk viscosity in Fig. 1. we obtain a clear correlation between the two ${ }^{13}$, with a first peak associated to the explicit breaking of the scale symmetry due to the finite quark mass, and a second peak near $T_{\mathrm{c}}$ due to the scale anomaly.

In the near future we plan to analyze carefully the massless case for the pion gas, vertex corrections in the calculation of the bulk viscosity, and the inclusion of finite pion-number chemical potential effects ${ }^{18}$.

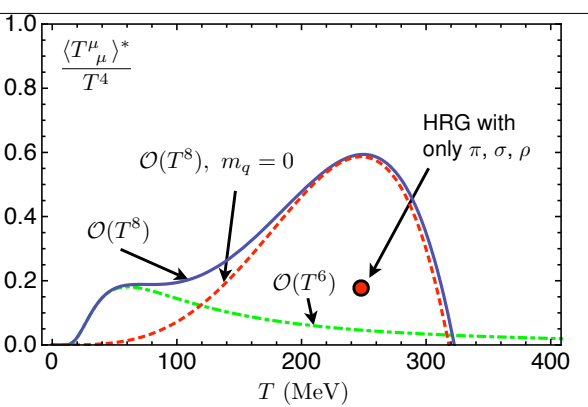

Fig. 2. Trace anomaly of the pion gas calculated in ChPT perturbatively.

The work of D.F.F. is sponsored by the Helmholtz International Center for FAIR. We both acknowledge financial support from the Spanish research Projects No. FPA2007-29115-E, No. PR34-1856-BSCH, No. UCM-BSCH GR58/08 910309, No. FPA2008-00592, No. FIS2008-01323.

\section{References}

1 D. T. Son and A. O. Starinets, Ann. Rev. Nucl. Part. Sci., 2007, 57: 95 .

2 G. Aarts, Nucl. Phys. A, 2009, 820: 57C.

3 D. Fernandez-Fraile and A. Gomez Nicola, Eur. Phys. J. C, 2009, 62: 37.

4 S. Jeon, Phys. Rev. D, 1995, 52: 3591.

5 S. Jeon and L. G. Yaffe, Phys. Rev. D, 1996, 53: 5799.

6 P. Arnold, G. D. Moore and L. G. Yaffe, JHEP, 2000, 0011: 001.

7 P. Arnold, C. Dogan and G. D. Moore, Phys. Rev. D, 2006, 74: 085021.

8 D. Fernandez-Fraile and A. Gomez Nicola, Phys. Rev. D, 2006, 73: 045025.

9 D. Fernandez-Fraile, A. Gomez Nicola and E. T. Herruzo,
Phys. Rev. D, 2007, 76: 085020.

10 D. Cabrera, D. Fernandez-Fraile and A.Gomez Nicola, Eur. Phys. J. C, 2009, 61: 879.

11 M. Prakash, M. Prakash, R. Venugopalan and G. Welke, Phys. Rept., 1993, 227: 321.

12 J. Noronha-Hostler, J. Noronha and C. Greiner, Phys. Rev. Lett., 2009, 103: 172302.

13 D. Fernandez-Fraile and A. Gomez Nicola, Phys. Rev. Lett., 2009, 102: 121601.

14 J. W. Chen and J. Wang, Phys. Rev. C, 2009, 79: 044913.

15 D. Kharzeev and K. Tuchin, JHEP, 2008, 0809: 093.

16 P. Romatschke and D. T. Son, Phys. Rev. D, 2009, 80: 065021.

17 S. Caron-Huot, Phys. Rev. D, 2009, 79: 125009.

18 D. Fernandez-Fraile and A. Gomez Nicola, Phys. Rev. D, 2009, 80: 056003. 